\title{
Note about T-duality of non-relativistic string
}

\section{J. Klusoň}

Department of Theoretical Physics and Astrophysics, Faculty of Science, Masaryk University, Kotlárská 2, 611 37, Brno, Czech Republic

E-mail: klu@physics.muni.cz

ABSTRACT: In this note we perform canonical analysis of T-duality for non-relativistic string in stringy Newton-Cartan background. We confirm recent result that T-duality along longitudinal spatial direction of stringy Newton-Cartan geometry maps non-relativistic string to the relativistic string that propagates on the background with light-like isometry.

Keywords: Bosonic Strings, String Duality

ARXIV EPrint: 1811.12658 


\section{Contents}

1 Introduction and summary 1

2 Review of stringy Newton-Cartan geometry 2

2.1 Hamiltonian for string in stringy Newton-Cartan geometry 4

3 T-duality in canonical formalism $\quad 6$

$\begin{array}{ll}3.1 \text { Longitudinal spatial T-duality transformation } & 6\end{array}$

4 Longitudinal lightlike T-duality $\quad 10$

5 Transverse T-duality 12

\section{Introduction and summary}

Recently, a very interesting non-relativistic string sigma model on stringy Newton Cartan geometry was proposed in [1]. ${ }^{1}$ This is ultraviolet finite theory which provides a quantization of stringy Newton-Cartan geometry in the same way as relativistic string theory provides quantum description of General Relativity. This model is generalization of the non-relativistic string theories that were proposed in $[4,5]$. These theories are invariant under string Galilean global symmetry and their characteristic (and crucial) property is an existence of two additional world-sheet fields beyond those parameterizing target space coordinates. The non-relativistic string sigma model proposed in [1] couples nonrelativistic string to background fields which are vielbein fields, Kalb-Ramond two form field and dilaton.

Another very interesting result that was found in [1] is related to the T-duality of non-relativistic string sigma model on an arbitrary string Newton-Cartan background. Due to the foliation of stringy Newton-Cartan structure there are two distinct T-duality transformations-transverse and longitudinal. These T-duality transformations were analyzed in the context of Lagrangian formalism with interesting results derived. It was shown that in the case of T-duality along longitudinal spatial direction we find world-sheet theory that corresponds to relativistic string propagating on Riemannian manifold with a compact lightlike isometry. This is a very intriguing result with possible important application for definition of discrete light cone quantization (DLCQ) of string theory. More precisely, DLCQ is defined as a quantization of theory compactified on light-like circle. In practise this is performed with the subtle limit of compactification on space-like circle [8-10]. Then we can hope that the result derived in [1] could be useful for an alternative definition of DLCQ of string theory on arbitrary Lorentzian background with lightlike isometry.

\footnotetext{
${ }^{1}$ For alternative approach to non-relativistic string in Newton-Cartan background see two recent interesting papers $[2,3]$.
} 
This fact is a very promising since it opens a new intriguing direction in the study of M-theory and its DLCQ description [6-8]. For that reason we mean that T-duality in the context of non-relativistic string theory on stringy Newton-Cartan background deserves further study. This is the goal of this paper when we would like to give a canonical description of T-duality of non-relativistic string. In fact, it is well known that in case of relativistic string, T-duality can be also interpreted as canonical transformation [11, 12]. In this paper we show that the same is true in case of non-relativistic string theory on stringy Newton-Cartan background. In order to do it we have to use the Hamiltonian form of the model [1] that it was found in [13]. With the help of this Hamiltonian we define T-duality as a canonical transformation. We focus on two physically different T-dualities: T-duality in longitudinal spatial direction and T-duality in transverse direction which have the same description in the canonical formalism. Performing these T-duality transformations we find T-dual Hamiltonian. Then in order to find explicit form of the transformed background field we derive corresponding Lagrangian and we find agreement with [1] in case of longitudinal spatial and transverse T-duality transformations. Explicitly, we find that T-duality along longitudinal spatial direction leads to T-dual relativistic string on the background with isometry in light-like direction while in case of T-duality in transverse direction we obtain again non-relativistic string in T-dual background. We mean that this is a very nice result that again shows how canonical treatment of T-duality transformation can be powerful.

We also discuss canonical description of another T-duality transformations that were analysed in [1]. The first one is longitudinal light like T-duality and we show that nonrelativistic string is mapped into non-relativistic string with light-like isometry. However the precise matching between canonical and Lagrangian description of this transformation is achieved when we perform redefinition of the Lagrange multipliers that are presented in the action for non-relativistic string [1]. Similar situation was previously observed in [1] in case of the longitudinal spatial T-duality where relativistic string with light-like isometry is mapped under T-duality to different non-relativistic strings that are related by redefinition of Lagrange multipliers. Finally we discuss transverse T-duality transformation where an isometry is along transverse spatial direction. In this case non-relativistic string is mapped under T-duality to non-relativistic string with agreement with [1].

The structure of this paper is as follows. In the next section (2) we review basic facts about stringy Newton-Cartan background and string sigma model together with its canonical formulation. In section (3) we introduce T-duality as canonical transformation, following $[11,12]$ and perform T-duality transformation along longitudinal spatial direction and determine background fields. In section (4) we perform T-duality along longitudinal light-like isometry and we analyze its properties. Finally in section (5) we perform canonical analysis of transverse T-duality.

\section{Review of stringy Newton-Cartan geometry}

Let us define string Newton-Cartan geometry following [1]. Let $\mathcal{M}$ is $D+1$ dimensional manifold and let $\mathcal{T}_{p}$ is tangent space at point $p$. We decompose $\mathcal{T}_{p}$ into longitudinal directions indexed by $A=0,1$ and transverse directions with $A^{\prime}=2, \ldots, d-1$. Two dimensional 
foliation of $\mathcal{M}$ is defined by generalized clock function $\tau_{\mu}{ }^{A}$ that is also known as longitudinal vielbein field that satisfies a constraint

$$
D_{\mu} \tau_{\nu}^{A}-D_{\nu} \tau_{\mu}^{A}=0
$$

where $D_{\mu}$ is covariant derivative with respect to the longitudinal Lorentz transformations acting on index $A$. Let us also introduce transverse vielbein field $E_{\mu}{ }^{A^{\prime}}$. We further introduce projective inverse $\tau_{A}^{\mu}$ and $E_{A^{\prime}}^{\mu}$ that obey following relations

$$
E_{\mu}^{A^{\prime}} E_{B^{\prime}}^{\mu}=\delta_{B^{\prime}}^{A^{\prime}}, \quad \tau_{A}^{\mu} \tau_{\mu}^{B}=\delta_{A}^{B}
$$

and also

$$
\tau_{\mu}{ }^{A} \tau_{A}^{\nu}+E_{\mu}^{A^{\prime}} E^{\nu}{ }_{A^{\prime}}=\delta_{\mu}^{\nu}, \quad \tau_{A}^{\mu} E_{\mu}^{A^{\prime}}=0, \quad \tau_{\mu}{ }^{A} E_{A^{\prime}}^{\mu}=0
$$

From $\tau_{\mu}{ }^{A}$ we can construct longitudinal metric $\tau_{\mu \nu}=\tau_{\mu}{ }^{A} \tau_{\nu}{ }^{B} \eta_{A B}$ and transverse metric $H^{\mu \nu}=E_{A^{\prime}}^{\mu} E_{B^{\prime}}^{\nu} \delta^{A^{\prime} B^{\prime}}$.

It is clear that in order to define string moving in stringy Newton-Cartan background we need tensor $H_{\mu \nu}$. It was shown in [1] that such a tensor has the form

$$
H_{\mu \nu}=E_{\mu}^{A^{\prime}} E_{\nu}^{B^{\prime}} \delta_{A^{\prime} B^{\prime}}+\left(\tau_{\mu}{ }^{A} m_{\nu}{ }^{B}+\tau_{\nu}{ }^{A} m_{\mu}{ }^{B}\right) \eta_{A B} .
$$

Now we are ready to write an action for non-relativistic string in this background [1]. It turns out that this action contains world-sheet scalars $x^{\mu}$ that parameterize an embedding string into target space time together with two additional world-sheet fields that we denote as $\lambda$ and $\bar{\lambda}$. These fields are needed for the realization of string Galilei symmetry on the world-sheet theory.

Now we will be more explicit. Let $\sigma^{\alpha}, \sigma^{0} \equiv \tau, \sigma^{1} \equiv \sigma$ parameterize world-sheet surface $\Sigma$. The sigma model is endowed with two dimensional world-sheet metric $h_{\alpha \beta}$ and we introduce two dimensional vielbein $e_{\alpha}^{a}, a=0,1$ so that

$$
h_{\alpha \beta}=e_{\alpha}^{a} e_{\beta}^{b} \eta_{a b} .
$$

Using light cone coordinates for the flat index $a$ on the world-sheet tangent space we define

$$
e_{\alpha} \equiv e_{\alpha}^{0}+e_{\alpha}^{1}, \quad \bar{e}_{\alpha} \equiv e_{\alpha}^{0}-e_{\alpha}^{1} .
$$

We can also use light-cone coordinates for the flat index $A$ on the space-time tangent space $\mathcal{T}_{p}$ and define

$$
\tau_{\mu} \equiv \tau_{\mu}^{0}+\tau_{\mu}^{1}, \bar{\tau}_{\mu}=\tau_{\mu}^{0}-\tau_{\mu}^{1} .
$$

Then we are ready to write sigma model for non-relativistic string on an arbitrary string Newton-Cartan geometry, NSNS two form and dilaton background in the form

$$
\begin{aligned}
S= & -\frac{T}{2} \int d^{2} \sigma\left(\sqrt{-h} h^{\alpha \beta} \partial_{\alpha} x^{\mu} \partial_{\beta} x^{\nu} H_{\mu \nu}+\epsilon^{\alpha \beta}\left(\lambda e_{\alpha} \tau_{\mu}+\bar{\lambda} \bar{e}_{\alpha} \bar{\tau}_{\mu}\right) \partial_{\beta} x^{\mu}\right) \\
& -\frac{T}{2} \int d^{2} \sigma \epsilon^{\alpha \beta} \partial_{\alpha} x^{\mu} \partial_{\beta} x^{\nu} B_{\mu \nu}+\frac{1}{4 \pi} \int d^{2} \sigma \sqrt{-h} R \Phi
\end{aligned}
$$


where $h=\operatorname{det} h_{\alpha \beta}, h^{\alpha \beta}$ is inverse to $h_{\beta \alpha}, R$ is scalar curvature of $h_{\alpha \beta}$ and $T$ is string tension. In what follows we restrict to the case of constant dilaton field so that the last term on the second line is total derivative and will be ignored. In other words, since we restrict ourselves to the classical canonical analysis we cannot determine transformation rules for dilaton under T-duality transformations.

\subsection{Hamiltonian for string in stringy Newton-Cartan geometry}

In our previous work [13] we performed Hamiltonian analysis of string in stringy Newton background. We found that the Hamiltonian is sum of two first class constraints $\mathcal{H}_{\tau}, \mathcal{H}_{\sigma}$ that have the form

$$
\begin{aligned}
\mathcal{H}_{\tau}= & \frac{1}{T} \pi_{\mu} H^{\mu \nu} \pi_{\nu}+T x^{\prime \mu} H_{\mu \nu} x^{\prime \nu}-\pi_{\mu} H^{\mu \nu}\left(\lambda^{+} \tau_{\nu}+\lambda^{-} \bar{\tau}_{\nu}\right) \\
& +T x^{\prime \mu}\left(\lambda^{+} \tau_{\mu}-\lambda^{-} \bar{\tau}_{\mu}\right)+\frac{T}{4}\left(\lambda^{+} \tau_{\mu}+\lambda^{-} \bar{\tau}_{\mu}\right) H^{\mu \nu}\left(\lambda^{+} \tau_{\nu}+\lambda^{-} \tau_{\nu}\right), \\
\mathcal{H}_{\sigma}= & x^{\prime \mu} p_{\mu}, \quad \pi_{\mu}=p_{\mu}+T B_{\mu \rho} x^{\prime \rho}, \quad x^{\prime \mu} \equiv \partial_{\sigma} x^{\mu},
\end{aligned}
$$

where the matrix $H^{\mu \nu}$ is inverse to $H_{\mu \nu}$ so that $H_{\mu \nu} H^{\nu \rho}=\delta_{\mu}^{\rho}$ with following explicit form

$$
H^{\mu \nu} \equiv h^{\mu \nu}-\hat{\tau}_{A}^{\mu}\left(\Phi^{-1}\right)^{A B} \hat{\tau}_{B}^{\nu}, \quad H^{\mu \nu} H_{\nu \rho}=\delta_{\rho}^{\mu},
$$

where we introduced $\hat{\tau}_{A}^{\mu}$ defined as

$$
\hat{\tau}_{A}^{\mu}=\tau_{A}^{\mu}-h^{\mu \rho} m_{\rho}^{B} \eta_{B A}
$$

Further, we also defined $2 \times 2$ matrix $\left(\Phi^{-1}\right)^{A B}$ with following explicit form

$$
\left(\Phi^{-1}\right)^{A B}=\frac{1}{\operatorname{det} \Phi_{A B}}\left(\begin{array}{cc}
\Phi_{11} & -\Phi_{01} \\
-\Phi_{01} & \Phi_{00}
\end{array}\right) .
$$

Clearly $\left(\Phi^{-1}\right)^{A B}$ is the matrix inverse to the matrix valued Newton potential that is defined as

$$
\Phi_{A B}=-\tau_{A}^{\sigma} m_{\sigma}^{C} \eta_{C B}-\eta_{A C} m_{\rho}^{C} \tau_{B}^{\rho}+\eta_{A C} m_{\rho}^{C} h^{\rho \sigma} m_{\sigma}{ }^{D} \eta_{D B} .
$$

Finally, $\lambda^{+}, \lambda^{-}$are two world-sheet scalar fields which are related to $\lambda, \bar{\lambda}$, for more details see [13]. Since $\lambda^{+}, \lambda^{-}$are non-dynamical their conjugate momenta are primary constraints and the requirement of their preservation implies an existence of two secondary constraints in the form

$$
\begin{aligned}
& \mathcal{G}_{+}^{\lambda}=\pi_{\mu} H^{\mu \nu} \tau_{\nu}-T x^{\prime \mu} \tau_{\mu}-\frac{T}{2} \tau_{\mu} H^{\mu \nu}\left(\lambda^{+} \tau_{\nu}+\lambda^{-} \bar{\tau}_{\nu}\right) \approx 0, \\
& \mathcal{G}_{-}^{\lambda}=\pi_{\mu} H^{\mu \nu} \bar{\tau}_{\nu}+T x^{\prime \mu} \bar{\tau}_{\mu}-\frac{T}{2} \bar{\tau}_{\mu} H^{\mu \nu}\left(\lambda^{+} \tau_{\nu}+\lambda^{-} \bar{\tau}_{\nu}\right) \approx 0 .
\end{aligned}
$$

These constraints are second class constraints together with momenta conjugate to $\lambda^{+}, \lambda^{-}$. Then it was shown in [13] that these constraints can be solved for $\lambda^{+}$and $\lambda^{-}$. Plugging 
these solutions to the original Hamiltonian constraint we obtain Hamiltonian constraint corresponding to the non-relativistic string action that was proposed in [14].

Finally we should also say few words about redefinition of the Lagrange multiplicators $\lambda^{+}, \lambda^{-}$. The best way how to perform this analysis is to switch to the Lagrangian formalism of non-relativistic string action. In fact, with the help of (2.9) we obtain

$$
\partial_{\tau} x^{\mu}=\frac{2 N}{T} H^{\mu \nu} \pi_{\nu}-H^{\mu \nu}\left(\lambda^{+} \tau_{\nu}+\lambda^{-} \bar{\tau}_{\nu}\right)+N^{\sigma} \partial_{\sigma} x^{\mu}
$$

and hence Lagrangian density has the form

$$
\begin{aligned}
\mathcal{L}= & p_{\mu} \partial_{\tau} x^{\mu}-N \mathcal{H}_{\tau}-N^{\sigma} \mathcal{H}_{\sigma} \\
= & \frac{N T}{4} \nabla_{n} x^{\mu} H_{\mu \nu} \nabla_{n} x^{\nu}+\frac{N T}{2} \nabla_{n} x^{\mu}\left(\lambda^{+} \tau_{\mu}+\lambda^{-} \bar{\tau}_{\mu}\right)-T N \partial_{\sigma} x^{\mu} H_{\mu \nu} \partial_{\sigma} x^{\nu} \\
& -T N \partial_{\sigma} x^{\mu}\left(\lambda^{+} \tau_{\mu}-\lambda^{-} \bar{\tau}_{\mu}\right)-T B_{\mu \nu} \partial_{\tau} x^{\mu} \partial_{\sigma} x^{\nu}, \\
\nabla_{n} x^{\mu}= & \frac{1}{N}\left(\partial_{\tau} x^{\mu}-N^{\sigma} \partial_{\sigma} x^{\mu}\right),
\end{aligned}
$$

where we used the fact that (2.15) implies

$$
\pi_{\mu}=\frac{T}{2} H_{\mu \nu} \nabla_{n} x^{\nu}+\frac{T}{2}\left(\lambda^{+} \tau_{\mu}+\lambda^{-} \bar{\tau}_{\mu}\right) .
$$

To see that the Lagrangian defines non-relativistic string theory let us solve the equation of motion for $\lambda^{+}$and $\lambda^{-}$respectively

$$
\nabla_{n} x^{\mu} \tau_{\mu}-2 \partial_{\sigma} x^{\mu} \tau_{\mu}=0, \quad \nabla_{n} x^{\mu} \bar{\tau}_{\mu}+2 \partial_{\sigma} x^{\mu} \bar{\tau}_{\mu}=0
$$

If we multiply the first equation with $\bar{\tau}_{\mu} \partial_{\sigma} x^{\mu}$ and the second one with $\tau_{\nu} \partial_{\sigma} x^{\nu}$ and sum them we obtain

$$
\nabla_{n} x^{\mu} \tau_{\mu} \bar{\tau}_{\nu} \partial_{\sigma} x^{\nu}+\nabla_{n} x^{\mu} \bar{\tau}_{\mu} \tau_{\nu} \partial_{\sigma} x^{\nu}=0
$$

that can be solved for $N^{\sigma}$ as

$$
N^{\sigma}=\frac{\tau_{\tau \sigma}}{\tau_{\tau \tau}}, \quad \tau_{\alpha \beta}=\tau_{\mu}{ }^{A} \tau_{\nu}{ }^{B} \eta_{A B} \partial_{\alpha} x^{\mu} \partial_{\beta} x^{\nu} .
$$

On the other hand when two equations given in (2.19) multiply together and use (2.20) we obtain

$$
N=\frac{\sqrt{-\operatorname{det} \tau}}{2 \tau_{\sigma \sigma}} .
$$

Inserting these results into (2.16) we finally obtain

$$
S=-\frac{T}{2} \sqrt{-\operatorname{det} \tau_{\alpha \beta}} \tau^{\alpha \beta} H_{\alpha \beta}-T B_{\mu \nu} \partial_{\tau} x^{\mu} \partial_{\sigma} x^{\nu},
$$

where $\tau^{\alpha \beta}$ is matrix inverse to $\tau_{\alpha \beta}$ and $H_{\alpha \beta}=H_{\mu \nu} \partial_{\alpha} x^{\mu} \partial_{\beta} x^{\nu}$. The Lagrangian density (2.22) is Nambu-Gotto formulation of non-relativistic string theory, see for example $[1,4]$. 
Let us now return to the Lagrangian density (2.16) and perform following redefinition of world-sheet modes $\lambda^{+}, \lambda^{-}$

$$
\begin{aligned}
& \lambda^{+}=C \tilde{\lambda}^{+}+\nabla_{n} x^{\mu} \mathbf{Y}_{\mu}^{+}+2 \partial_{\sigma} x^{\mu} \mathbf{Y}_{\mu}^{+}, \\
& \lambda^{-}=C \tilde{\lambda}^{-}+\nabla_{n} x^{\mu} \mathbf{Y}_{\mu}^{-}-2 \partial_{\sigma} x^{\mu} \mathbf{Y}_{\mu}^{-},
\end{aligned}
$$

where $C, \mathbf{Y}_{\mu}^{+}, \mathbf{Y}_{\mu}^{-}$are arbitrary functions. Under such a redefinition the Lagrangian density (2.16) takes equivalent form

$$
\begin{aligned}
\mathcal{L}= & \frac{N T}{4} \nabla_{n} x^{\mu} H_{\mu \nu}^{R} \nabla_{n} x^{\nu}+\frac{N T}{2} \nabla_{n} x^{\mu}\left(\tilde{\lambda}^{+} \tau_{\mu}^{R}+\tilde{\lambda}^{-} \bar{\tau}_{\mu}^{R}\right)-T N \partial_{\sigma} x^{\mu} H_{\mu \nu}^{R} \partial_{\sigma} x^{\nu} \\
& -T N \partial_{\sigma} x^{\mu}\left(\tilde{\lambda}^{+} \tau_{\mu}^{R}-\tilde{\lambda}^{-} \bar{\tau}_{\mu}^{R}\right)-T B_{\mu \nu}^{R} \partial_{\tau} x^{\mu} \partial_{\sigma} x^{\nu}
\end{aligned}
$$

where we have following background fields

$$
\begin{aligned}
\tau_{\mu}^{R} & =C \tau_{\mu}, \quad \bar{\tau}_{\mu}^{R}=C \bar{\tau}_{\mu} \\
H_{\mu \nu}^{R} & =H_{\mu \nu}+\tau_{\mu} \mathbf{Y}_{\nu}^{+}+\mathbf{Y}_{\mu}^{+} \tau_{\nu}+\bar{\tau}_{\mu} \mathbf{Y}_{\nu}^{-}+\mathbf{Y}_{\mu}^{-} \bar{\tau}_{\nu} \\
B_{\mu \nu}^{R} & =B_{\mu \nu}-\tau_{\mu} \mathbf{Y}_{\nu}^{+}-\mathbf{Y}_{\mu}^{+} \tau_{\nu}+\bar{\tau}_{\mu} \mathbf{Y}_{\nu}^{-}-\mathbf{Y}_{\mu}^{-} \bar{\tau}_{\nu}
\end{aligned}
$$

In the next section we will analyze the question how these Lagrangian densities for nonrelativistic strings which are related by redefinition of the Lagrange multipliers map under longitudinal spatial T-duality transformations.

\section{T-duality in canonical formalism}

In this section we present canonical analysis of T-duality for non-relativistic string. Due to the non-trivial foliation of target space-time we have to distinguish between T-duality transformations in the longitudinal and transverse directions. However we will see that these two transformations have the same treatment in the canonical formalism. Let us start our analysis with longitudinal spatial T-duality transformation.

\subsection{Longitudinal spatial T-duality transformation}

Following [1] we presume that non-relativistic theory possesses longitudinal spatial Killing vector $k^{\mu}$ that obeys the relation

$$
\tau_{\mu}^{0} k^{\mu}=0, \quad \tau_{\mu}^{1} k^{\mu} \neq 0, \quad E_{\mu}^{A^{\prime}} k^{\mu}=0
$$

It is convenient to introduce coordinate system $\left(y, x^{i}\right)$ adapted to $k^{\mu}$ such that $k^{\mu} \partial_{\mu}=$ $\partial_{y}$. It is important to stress that $x^{i}$ contains longitudinal coordinate. Then the previous condition implies

$$
\tau_{y}^{0}=0, \quad \tau_{y}{ }^{1} \neq 0, \quad E_{y}^{A^{\prime}}=0, \quad \tau_{y}=-\bar{\tau}_{y} \neq 0 .
$$

Finally, in adapted coordinates all background fields are independent on $y$. As a result the theory is invariant under shift

$$
y^{\prime} \rightarrow y+\epsilon, \epsilon=\text { const }
$$


Our goal is to perform canonical transformation from $y$ to $\tilde{y}$ in the same way as in case of relativistic string $[11,12]$. As was shown there the generating function has the form

$$
G(y, \tilde{y})=\frac{T}{2} \int d \sigma\left(\partial_{\sigma} y \tilde{y}-y \partial_{\sigma} \tilde{y}\right) .
$$

Let us denote momentum conjugate to $\tilde{y}$ as $p_{\tilde{y}}$. Then from the definition of the canonical transformations we derive following relation between $\tilde{y}$ and $p_{\tilde{y}}$ in the form

$$
\begin{aligned}
& p_{\tilde{y}}=-\frac{\delta G}{\delta \tilde{y}}=-T \partial_{\sigma} y, \\
& p_{y}=\frac{\delta G}{\delta y}=-T \partial_{\sigma} \tilde{y} .
\end{aligned}
$$

With the help of these relations we obtain dual Hamiltonian when we replace $\partial_{\sigma} y$ with $-\frac{1}{T} p_{\tilde{y}}$ and $p_{y}$ with $-T \partial_{\sigma} y$. Explicitly, using the constraints given in (2.9) we obtain Tdual constraints in the form

$$
\begin{aligned}
\mathcal{H}_{\tau}^{T}= & \frac{1}{T}\left(k_{i}-B_{i y} p_{\tilde{y}}\right) H^{i j}\left(k_{j}-B_{j y} p_{\tilde{y}}\right)+\frac{2}{T}\left(-T \tilde{y}^{\prime}+T B_{y k} x^{\prime k}\right) H^{y i}\left(k_{i}-B_{i \tilde{y}} p_{\tilde{y}}\right) \\
& +\frac{1}{T}\left(-T \tilde{y}^{\prime}+T B_{y i} x^{i}\right) H^{y y}\left(-T \tilde{y}^{\prime}+T B_{y j} x^{\prime j}\right)+\frac{1}{T} p_{\tilde{y}} H_{y y} p_{\tilde{y}}-2 p_{\tilde{y}} H_{y j} x^{\prime j}+T x^{\prime i} H_{i j} x^{\prime j} \\
& -\left(-T \tilde{y}^{\prime}+T B_{y i} x^{i}\right) H^{y y}\left(\lambda^{+} \tau_{y}+\lambda^{-} \bar{\tau}_{y}\right)-\left(-T \tilde{y}^{\prime}+T B_{y i} x^{i}\right) H^{y j}\left(\lambda^{+} \tau_{j}+\lambda^{-} \bar{\tau}_{j}\right) \\
& -\left(k_{i}-B_{i y} p_{\tilde{y}}\right) H^{i j}\left(\lambda^{+} \tau_{j}+\lambda^{-} \bar{\tau}_{j}\right)-\left(k_{i}-B_{i y} p_{\tilde{y}}\right) H^{i y}\left(\lambda^{+} \tau_{y}+\lambda^{-} \bar{\tau}_{y}\right) \\
& +T x^{\prime i}\left(\lambda^{+} \tau_{i}-\lambda^{-} \bar{\tau}_{i}\right)-p_{\tilde{y}}\left(\lambda^{+} \tau_{y}-\lambda^{-} \bar{\tau}_{y}\right)+\frac{T}{4}\left(\lambda^{+} \tau_{\mu}+\lambda^{-} \bar{\tau}_{\mu}\right) H^{\mu \nu}\left(\lambda^{+} \tau_{\nu}+\lambda^{-} \bar{\tau}_{\nu}\right), \\
\mathcal{H}_{\sigma}^{T}= & x^{i i} p_{i}+\tilde{y}^{\prime} p_{\tilde{y}},
\end{aligned}
$$

where $k_{i}=p_{i}+T B_{i j} \partial_{\sigma} x^{j}$. We see that it is very difficult to find T-dual background fields from this form of the Hamiltonian which is a consequence of the fact that symmetries are usually hidden in the canonical formalism. On the other hand symmetric structures and forms of the background fields naturally emerge in the Lagrangian formalism so that we should determine Lagrangian density corresponding to T-dual constraints (3.6). To do this we determine time evolution of $x^{i}$ and $\tilde{y}$ using T-dual Hamiltonian in the form

$$
H^{T}=\int d \sigma\left(N \mathcal{H}_{\tau}^{T}+N^{\sigma} \mathcal{H}_{\sigma}^{T}\right)
$$

where $\mathcal{H}_{\tau}^{T}, \mathcal{H}_{\sigma}^{T}$ are given in (3.6). Explicitly we obtain

$$
\begin{aligned}
\partial_{\tau} x^{i}=\left\{x^{i}, H^{T}\right\}= & \frac{2 N}{T} H^{i j}\left(k_{j}-B_{j y} p_{\tilde{y}}\right)+\frac{2 N}{T} H^{i y}\left(-T \tilde{y}^{\prime}+T B_{y j} x^{\prime j}\right) \\
& -N H^{i j}\left(\lambda^{+} \tau_{j}+\lambda^{-} \bar{\tau}_{j}\right)-N H^{i y}\left(\lambda^{+} \tau_{y}+\lambda^{-} \bar{\tau}_{y}\right)+N^{\sigma} x^{i}, \\
\partial_{\tau} \tilde{y}=\left\{\tilde{y}, H^{T}\right\}= & -\frac{2 N}{T} B_{i y} H^{i j}\left(k_{j}-B_{j y} p_{\tilde{y}}\right)-\frac{2 N}{T}\left(-T \tilde{y}^{\prime}+T B_{y j} x^{\prime j}\right) H^{y i} B_{i y} \\
& +\frac{2 N}{T} H_{y y} p_{\tilde{y}}-2 N H_{y j} x^{\prime j}+N B_{i y} H^{i j}\left(\lambda^{+} \tau_{j}+\lambda^{-} \bar{\tau}_{j}\right) \\
& +N B_{i y} H^{i y}\left(\lambda^{+} \tau_{y}+\lambda^{-} \bar{\tau}_{y}\right)-N\left(\lambda^{+} \tau_{y}-\lambda^{-} \bar{\tau}_{y}\right)+N^{\sigma} \tilde{y}^{\prime} .
\end{aligned}
$$


From the last equation we can express $p_{\tilde{y}}$ as

$$
p_{\tilde{y}}=\frac{T}{2 N H_{y y}}\left(\tilde{Y}+B_{j y} X^{j}+2 N H_{y j} x^{\prime j}+N\left(\lambda^{+} \tau_{y}-\lambda^{-} \bar{\tau}_{y}\right)\right)
$$

where we defined

$$
X^{i}=\dot{x}^{i}-N^{\sigma} x^{i}, \quad \tilde{Y}=\dot{\tilde{y}}-N^{\sigma} \tilde{y}^{\prime} .
$$

In case of $k_{i}$ the situation is more involved since we have to find metric inverse to $H^{i j}$. It turns out that it has the form

$$
h_{i j}=H_{i j}-\frac{H_{i y} H_{y j}}{H_{y y}} .
$$

For further purposes we write following relation

$$
h_{i j} H^{j y}=-\frac{H_{i y}}{H_{y y}}
$$

that follows from the definition of $h_{i j}$. With the help of the inverse metric we find corresponding Lagrangian density

$$
\begin{aligned}
\mathcal{L}^{T}= & p_{\tilde{y}} \partial_{\tau} \tilde{y}+p_{i} \partial_{\tau} x^{i}-H^{T} \\
= & \frac{T}{4 N}\left(\tilde{g}_{\tau \tau}-2 N^{\sigma} \tilde{g}_{\sigma \tau}+\left(N^{\sigma}\right)^{2} \tilde{g}_{\sigma \sigma}\right)-N T \tilde{g}_{\sigma \sigma}-T \tilde{B}_{\mu \nu} \partial_{\tau} \tilde{x}^{\mu} \partial_{\sigma} \tilde{x}^{\nu} \\
& +\frac{T}{2} \lambda^{+} A+\frac{T}{2} \lambda^{-} B+\frac{N T}{H_{y y}} \lambda^{+} \lambda^{-} \tau_{y} \tau_{y},
\end{aligned}
$$

where

$$
\tilde{g}_{\alpha \beta}=\partial_{\alpha} \tilde{x}^{\mu} \tilde{H}_{\mu \nu} \partial_{\beta} \tilde{x}^{\nu}, \quad \tilde{x}^{\mu}=\left(x^{i}, \tilde{y}\right),
$$

and where we have T-dual components of metric and NSNS two form

$$
\begin{array}{rlrl}
\tilde{H}_{y y} & =\frac{1}{H_{y y}}, & \tilde{H}_{i j}=H_{i j}-\frac{H_{i y} H_{y j}}{H_{y y}}+\frac{1}{H_{y y}} B_{i y} B_{j y}, \\
\tilde{H}_{y i}=\tilde{H}_{i y}=\frac{B_{i y}}{H_{y y}}, & \tilde{B}_{\tilde{y} i}=\frac{H_{y i}}{H_{y y}}, \\
\tilde{B}_{i j}=B_{i j}+\frac{B_{i y} H_{y j}}{H_{y y}}-\frac{H_{i y} B_{y j}}{H_{y y}}, & \\
\tilde{B}_{i \tilde{y}}=-\frac{H_{y i}}{H_{y y}} & &
\end{array}
$$

Note that these transformation components of background fields agree with known Buscher's rules $[15,16]$. The novelty of non-relativistic Lagrangian is the presence of terms on the last line in (3.13) where $A$ and $B$ are equal to

$$
\begin{aligned}
& A=\frac{1}{H_{y y}}\left(\dot{x}^{i} \mathbf{A}_{i}+\dot{\tilde{y}} \tau_{y}-N^{\sigma}\left[x^{i} \mathbf{A}_{i}+\tilde{y}^{\prime} \tau_{y}\right]-2 N\left[x^{i} \mathbf{A}_{i}+\tilde{y}^{\prime} \tau_{y}\right]\right), \\
& B=\frac{1}{H_{y y}}\left(\dot{x}^{i} \mathbf{B}_{i}-\dot{\tilde{y}} \bar{\tau}_{y}-N^{\sigma}\left[x^{\prime i} \mathbf{B}_{i}-\tilde{y}^{\prime} \bar{\tau}_{y}\right]+2 N\left[x^{i} \mathbf{B}_{i}-\tilde{y}^{\prime} \bar{\tau}_{y}\right]\right),
\end{aligned}
$$


where $\mathbf{A}_{\mu}$ and $\mathbf{B}_{\mu}$ are equal to

$$
\begin{array}{ll}
\mathbf{A}_{i}=H_{y y} \tau_{i}+B_{i y} \tau_{y}-H_{i y} \tau_{y}, & \mathbf{A}_{y}=\tau_{y}, \\
\mathbf{B}_{i}=H_{y y} \bar{\tau}_{i}-B_{i y} \bar{\tau}_{y}-H_{i y} \bar{\tau}_{y}, & \mathbf{B}_{y}=-\bar{\tau}_{y} .
\end{array}
$$

As the last step we will solve the equations of motion for $\lambda^{+}$and $\lambda^{-}$that have the form

$$
\frac{1}{2} A+\frac{N}{H_{y y}} \lambda^{-} \tau_{y} \tau_{y}=0, \quad \frac{1}{2} B+\frac{N}{H_{y y}} \lambda^{+} \tau_{y} \tau_{y}=0
$$

with corresponding solutions

$$
\lambda^{-}=-\frac{H_{y y}}{2 N} \frac{A}{\tau_{y} \tau_{y}}, \quad \lambda^{+}=-\frac{H_{y y}}{2 N} \frac{B}{\tau_{y} \tau_{y}} .
$$

Inserting back to the Lagrangian density (3.13) we obtain

$$
\mathcal{L}^{T}=\frac{T}{4 N}\left(\tilde{g}_{\tau \tau}-2 N^{\sigma} \tilde{g}_{\sigma \tau}+\left(N^{\sigma}\right)^{2} \tilde{g}_{\sigma \sigma}\right)-N T \tilde{g}_{\sigma \sigma}-T \tilde{B}_{\mu \nu} \partial_{\tau} \tilde{x}^{\mu} \partial_{\sigma} \tilde{x}^{\nu}-\frac{1}{4 N} T \frac{H_{y y}}{\tau_{y} \tau_{y}} A B
$$

where

$$
\begin{aligned}
-\frac{H_{y y}}{4 N \tau_{y} \tau_{y}} A B= & -\frac{1}{4 N \tau_{y} \tau_{y} H_{y y}}\left[\frac{1}{2} \partial_{\tau} \tilde{x}^{\mu} \partial_{\tau} \tilde{x}^{\nu}\left(\mathbf{A}_{\mu} \mathbf{B}_{\nu}+\mathbf{A}_{\nu} \mathbf{B}_{\mu}\right)\right. \\
& -2 N^{\sigma} \partial_{\tau} \tilde{x}^{\mu} \partial_{\sigma} \tilde{x}^{\nu} \frac{1}{2}\left(\mathbf{A}_{\mu} \mathbf{B}_{\nu}+\mathbf{A}_{\nu} \mathbf{B}_{\mu}\right)+2 N \partial_{\tau} \tilde{x}^{\mu} \partial_{\sigma} \tilde{x}^{\nu}\left(\mathbf{A}_{\mu} \mathbf{B}_{\nu}-\mathbf{A}_{\nu} \mathbf{B}_{\mu}\right) \\
& +\frac{1}{2}\left(N^{\sigma}\right)^{2} \partial_{\sigma} \tilde{x}^{\mu} \partial_{\sigma} \tilde{x}^{\nu}\left(\mathbf{A}_{\mu} \mathbf{B}_{\nu}+\mathbf{A}_{\nu} \mathbf{B}_{\mu}\right) \\
& \left.-4 N^{2} \partial_{\sigma} \tilde{x}^{\mu} \partial_{\sigma} \tilde{x}^{\nu} \frac{1}{2}\left(\mathbf{A}_{\mu} \mathbf{B}_{\nu}+\mathbf{A}_{\nu} \mathbf{B}_{\mu}\right)\right]
\end{aligned}
$$

Then using explicit form of $\mathbf{A}_{\mu}, \mathbf{B}_{\mu}$ given in (3.17) we obtain final form of the Lagrangian density

$$
\mathcal{L}^{T}=\frac{T}{4 N}\left(g_{\tau \tau}^{\prime}-2 N^{\sigma} g_{\sigma \tau}^{\prime}+\left(N^{\sigma}\right)^{2} g_{\sigma \sigma}^{\prime}\right)-N T g_{\sigma \sigma}^{\prime}-T B_{\mu \nu}^{\prime} \partial_{\tau} \tilde{x}^{\mu} \partial_{\sigma} \tilde{x}^{\nu}
$$

where

$$
g_{\alpha \beta}^{\prime}=H_{\mu \nu}^{\prime} \partial_{\alpha} \tilde{x}^{\mu} \partial_{\beta} \tilde{x}^{\nu}
$$

where

$$
\begin{aligned}
& H_{i j}^{\prime}=H_{i j}+\frac{H_{y y}}{\tau_{y y}} \tau_{i j}-\frac{\tau_{i y}}{\tau_{y y}} H_{j y}-\frac{\tau_{j y}}{\tau_{y y}} H_{i y}+\frac{1}{\tau_{y y}}\left(B y i \tau_{j}{ }^{A} \tau_{y}{ }^{B} \epsilon_{A B}+B B_{y i} \tau_{j}{ }^{A} \tau_{y}{ }^{B} \epsilon_{A B}\right), \\
& H_{\tilde{y} \tilde{y}}^{\prime}=0, \quad H_{\tilde{y} i}^{\prime}=-\frac{\tau_{i}{ }^{A} \tau_{y}{ }^{B} \epsilon_{A B}}{\tau_{y y}}
\end{aligned}
$$

where we have convention $\epsilon_{01}=-1, \epsilon_{10}=1$. Finally note that $B_{\mu \nu}^{\prime}$ is equal to

$$
\begin{aligned}
& B_{i j}^{\prime}=B_{i j}-\frac{H_{y y}}{\tau_{y} \tau_{y}} \tau_{i}^{A} \tau_{j}{ }^{B} \epsilon_{A B}+\frac{\tau_{i y} B_{y j}-B_{y i} \tau_{j y}}{\tau_{y} \tau_{y}}+\frac{\tau_{i}{ }^{A} \tau_{y}{ }^{B} \epsilon_{A B} H_{j y}-\tau_{j}{ }^{A} \tau_{y}{ }^{B} \epsilon_{A B} H_{i y}}{\tau_{y} \tau_{y}}, \\
& B_{i \tilde{y}}^{\prime}=-\frac{\tau_{i y}}{\tau_{y} \tau_{y}}
\end{aligned}
$$


These transformation rules agree with the result derived in [1]. We also see that the T-dual string corresponds to the relativistic string with light-like isometry due to the absence of the metric component $H_{\tilde{y} \tilde{y}}^{\prime}$. To see more explicitly that we have relativistic string let us solve the equations of motion for $N$ and $N^{\sigma}$ :

$$
-g_{\sigma \tau}^{\prime}+N^{\sigma} g_{\sigma \sigma}^{\prime}=0, \quad-\frac{1}{4 N^{2}}\left(g_{\sigma \sigma}^{\prime}-2 N^{\sigma} g_{\sigma \tau}^{\prime}+\left(N^{\sigma}\right)^{2} g_{\sigma \sigma}^{\prime}\right)-g_{\sigma \sigma}^{\prime}=0 .
$$

These equations can be solved for $N^{\sigma}$ and $N$ as

$$
N^{\sigma}=\frac{g_{\sigma \tau}^{\prime}}{g_{\sigma \sigma}^{\prime}}, \quad N=\frac{1}{2} \frac{\sqrt{-\operatorname{det} g_{\alpha \beta}^{\prime}}}{g_{\sigma \sigma}^{\prime}}
$$

Inserting back to $(3.22)$ we obtain

$$
\mathcal{L}^{T}=-T \sqrt{-\operatorname{det} g_{\alpha \beta}^{\prime}}-T B_{\mu \nu}^{\prime} \partial_{\tau} \tilde{x}^{\mu} \partial_{\sigma} \tilde{x}^{\nu}
$$

that is relativistic string action in Nambu-Goto form.

Apparently the same procedure can be performed with the Lagrangian density (2.24) where

$$
\begin{aligned}
H_{i j}^{\prime \prime}= & H_{i j}^{R}+\frac{H_{y y}^{R}}{\tau_{y y}^{R}} \tau_{i j}^{R}-\frac{\tau_{i y}^{R}}{\tau_{y y}^{R}} H_{j y}^{R}-\frac{\tau_{j y}^{R}}{\tau_{y y}^{R}} H_{i y}^{R} \\
& +\frac{1}{\tau_{y y}^{R}}\left(B_{y i}^{R}\left(\tau_{j}^{R}\right)^{A}\left(\tau_{y}^{R}\right)^{B} \epsilon_{A B}+B_{y j}^{R} \tau_{i}^{R, A} \tau_{y}^{R, B} \epsilon_{A B}\right), \\
H_{\tilde{y} \tilde{y}}^{\prime \prime}= & 0, \quad H_{\tilde{y} i}^{\prime \prime}=-\frac{\tau_{i}^{R},{ }^{A} \tau_{y}^{R},{ }^{B} \epsilon_{A B}}{\tau_{y y}^{R}} .
\end{aligned}
$$

Now with the help of $(2.25)$ it is easy to see that $H_{\mu \nu}^{\prime \prime}=H_{\mu \nu}^{\prime}$. In other words, relativistic string with light-like isometry is mapped to different Newton-Cartan backgrounds that are related by fields redefinition (2.25) which is in agreement with [1].

Let us now briefly discuss an inverse T-duality transformation from the relativistic action with light-like isometry to stringy Newton-Cartan background. The starting point is the relativistic string in the background (3.24) and (3.25). Then since the Lagrangian is linear in the coordinate along which T-duality should be performed it is convenient to start with equivalent Lagrangian density (3.13) that contain two auxiliary fields $\lambda^{+}, \lambda^{-}$ and the background fields given in (3.15) and with $A$ and $B$ given in (3.16). Clearly corresponding Hamiltonian is given in (3.6) and hence inverse T-duality rules (3.5) maps the Hamiltonian (3.6) to (2.9) that corresponds to the Hamiltonian constraint of nonrelativistic string on stringy Newton-Cartan background.

\section{Longitudinal lightlike T-duality}

In this section we study longitudinal lightlike T-duality transformation in the canonical formalism. In other words we study T-duality along light-like isometry direction. Let us presume that non-relativistic sigma model has lightlike killing vector $l^{\mu}$ that obeys

$$
\tau_{\mu} l^{\mu} \neq 0, \quad \bar{\tau}_{\mu} l^{\mu}=0, \quad E_{\mu}^{A^{\prime}} l^{\mu}=0 .
$$


We define coordinate system $\left(u, x^{i}\right)$ adapted to $l^{\mu}$ so that

$$
\tau_{u} \neq 0, \quad \bar{\tau}_{u}=0, \quad E_{u}^{A^{\prime}}=0 .
$$

Now performing T-duality according to (3.5) when we replace $y$ with $u$ and $\tilde{y}$ with $\tilde{u}$ we obtain Hamiltonian constraint in the form

$$
\begin{aligned}
\mathcal{H}_{\tau}^{T}= & \frac{1}{T}\left(k_{i}-B_{i u} p_{\tilde{u}}\right) H^{i j}\left(k_{j}-B_{j u} p_{\tilde{u}}\right)-\left(\tilde{u}^{\prime}-B_{u k} x^{\prime k}\right) H^{u i}\left(k_{i}-B_{i u} p_{\tilde{u}}\right) \\
& +T^{2}\left(\tilde{u}^{\prime}-B_{u i} x^{i}\right) H^{u u}\left(\tilde{u}^{\prime}-B_{u j} x^{\prime j}\right)+\frac{1}{T} p_{\tilde{u}} H_{u u} p_{\tilde{u}}-2 p_{\tilde{u}} H_{u i} x^{i}+T x^{i} H_{i j} x^{j} \\
& -\left(k_{i}-B_{i \tilde{u}} p_{\tilde{u}}\right) H^{i j}\left(\lambda^{+} \tau_{j}+\lambda^{-} \bar{\tau}_{j}\right)-\left(k_{i}-B_{i \tilde{u}} p_{\tilde{u}}\right) H^{i u} \lambda^{+} \tau_{u} \\
& +T\left(\tilde{u}^{\prime}-B_{u i} x^{\prime i}\right) H^{u u} \lambda^{+} \tau_{u}+T\left(\tilde{u}^{\prime}-B_{u i} x^{i}\right) H^{u j}\left(\lambda^{+} \tau_{j}+\lambda^{-} \bar{\tau}_{j}\right) \\
& +T x^{\prime i}\left(\lambda^{+} \tau_{i}-\lambda^{-} \bar{\tau}_{i}\right)-p_{\tilde{u}} \lambda^{+} \tau_{u}+\frac{T}{4}\left(\lambda^{+} \tau_{i}+\lambda^{-} \bar{\tau}_{i}\right) H^{i j}\left(\lambda^{+} \tau_{j}+\lambda^{-} \bar{\tau}_{j}\right) \\
& +\frac{T}{2}\left(\lambda^{+} \tau_{i}+\lambda^{-} \bar{\tau}_{i}\right) H^{i u} \lambda^{+} \tau_{u}
\end{aligned}
$$

so that T-dual Hamiltonian has the form

$$
H^{T}=\int d \sigma\left(N \mathcal{H}_{\tau}^{T}+N^{\sigma} \mathcal{H}_{\sigma}^{T}\right) .
$$

Then in the same way as in previous section we convert this Hamiltonian to the Lagrangian and we obtain

$$
\begin{aligned}
\mathcal{L}= & p_{i} \dot{x}^{i}+p_{\tilde{u}} \dot{\tilde{u}}-N \mathcal{H}_{\tau}^{T}-N^{\sigma} \mathcal{H}_{\sigma}^{T} \\
= & \frac{T}{4 N}\left(\tilde{g}_{\tau \tau}-2 N^{\sigma} \tilde{g}_{\tau \sigma}+\left(N^{\sigma}\right)^{2} \tilde{g}_{\sigma \sigma}^{2}\right)-N T \tilde{g}_{\sigma \sigma}-T \partial_{\tau} \tilde{x}^{\mu} \tilde{B}_{\mu \nu} \partial_{\sigma} \tilde{x}^{\nu} \\
& +\frac{T}{2 H_{u u}} \lambda^{+}\left(\partial_{\tau} \tilde{x}^{\mu} \mathbf{A}_{\mu}-N^{\sigma} \partial_{\sigma} \tilde{x}^{\mu} \mathbf{A}_{\mu}-2 N \partial_{\sigma} \tilde{x}^{\mu} \mathbf{A}_{\mu}\right) \\
& +\frac{T}{2 H_{u u}} \lambda^{-}\left(\partial_{\tau} \tilde{x}^{\mu} \mathbf{B}_{\mu}-N^{\sigma} \partial_{\sigma} \tilde{x}^{\mu} \mathbf{B}_{\mu}+2 N \partial_{\sigma} \tilde{x}^{\mu} \mathbf{B}_{\mu}\right)
\end{aligned}
$$

where again

$$
\tilde{g}_{\alpha \beta}=\tilde{G}_{\mu \nu} \partial_{\alpha} \tilde{X}^{\mu} \partial_{\beta} \tilde{X}^{\nu}
$$

and where

$$
\begin{aligned}
\tilde{G}_{i j}=H_{i j}-\frac{H_{i u} H_{u j}}{H_{u u}}-\frac{B_{i u} B_{u j}}{H_{u u}}, & \tilde{G}_{i \tilde{u}}=\frac{B_{i u}}{H_{u u}}, & \tilde{G}_{\tilde{u} \tilde{u}}=\frac{1}{H_{u u}}, \\
\tilde{B}_{i j}=B_{i j}+\frac{B_{u i} H_{u j}-H_{i u} B_{u j}}{H_{u u}}, & \tilde{B}_{i \tilde{u}}=\frac{H_{i u}}{H_{u u}}, & \tilde{B}_{\tilde{u} i}=-\frac{H_{u i}}{H_{u u}}, \\
\mathbf{A}_{i}=\tau_{i} H_{u u}-H_{i u} \tau_{u}+B_{i u} \tau_{u}, & \mathbf{A}_{\tilde{u}}=\tau_{u}, & \mathbf{B}_{i}=\bar{\tau}_{i} H_{u u} .
\end{aligned}
$$

In our special case we have $\bar{\tau}_{u}=0$ and hence T-dual string has the same form as nonrelativistic string in stringy Newton Cartan background. However it is important to stress 
that the values of the background fields are not uniquely determined by T-duality rules since it is possible to perform redefinition of $\lambda^{+}$and $\lambda^{-}$in the form

$$
\begin{aligned}
& \lambda^{+}=\frac{H_{u u}}{\tau_{u} \tau_{u}} \tilde{\lambda}^{+}+\frac{1}{N}\left(\partial_{\tau} \tilde{x}^{\mu} \mathbf{Y}_{\mu}^{+}-N^{\sigma} \partial_{\sigma} \tilde{x}^{\mu} \mathbf{Y}_{\mu}^{+}+2 N \partial_{\sigma} \tilde{x}^{\mu} \mathbf{Y}_{\mu}^{+}\right), \\
& \lambda^{-}=H_{u u} \tilde{\lambda}^{-}+\frac{1}{N}\left(\partial_{\tau} \tilde{x}^{\mu} \mathbf{Y}_{\mu}^{-}-N^{\sigma} \partial_{\sigma} \tilde{x}^{\mu} \mathbf{Y}_{\mu}^{-}-2 N \partial_{\sigma} \tilde{x}^{\mu} \mathbf{Y}_{\mu}^{-}\right)
\end{aligned}
$$

so that the Lagrangian density changes as

$$
\begin{aligned}
\mathcal{L}^{T}= & \frac{T}{4 N}\left(\tilde{g}_{\tau \tau}^{\prime}-2 N^{\sigma} \tilde{g}_{\tau \sigma}^{\prime}+\left(N^{\sigma}\right)^{2} \tilde{g}_{\sigma \sigma}^{\prime}\right)-N T \tilde{g}_{\sigma \sigma}^{\prime}-T \partial_{\tau} \tilde{x}^{\mu} \tilde{B}_{\mu \nu}^{\prime} \partial_{\sigma} \tilde{x}^{\nu} \\
& +\frac{T}{2} \tilde{\lambda}^{+}\left(\partial_{\tau} \tilde{x}^{\mu} \mathbf{A}_{\mu}-N^{\sigma} \partial_{\sigma} \tilde{x}^{\mu} \mathbf{A}_{\mu}-2 N \partial_{\sigma} \tilde{x}^{\mu} \mathbf{A}_{\mu}\right) \\
& +\frac{T}{2} \tilde{\lambda}^{-}\left(\partial_{\tau} \tilde{x}^{\mu} \mathbf{B}_{\mu}-N^{\sigma} \partial_{\sigma} \tilde{x}^{\mu} \mathbf{B}_{\mu}+2 N \partial_{\sigma} \tilde{x}^{\mu} \mathbf{B}_{\mu}\right),
\end{aligned}
$$

where

$$
g_{\alpha \beta}^{\prime}=\tilde{G}_{\mu \nu}^{\prime} \partial_{\alpha} \tilde{x}^{\mu} \partial_{\beta} \tilde{x}^{\nu},
$$

and where $\tilde{G}_{\mu \nu}^{\prime}$ and $\tilde{B}_{\mu \nu}^{\prime}$ have the form

$$
\begin{aligned}
& \tilde{G}_{\mu \nu}^{\prime}=\tilde{G}_{\mu \nu}+\frac{1}{H_{u u}}\left(\mathbf{A}_{\mu} \mathbf{Y}_{\nu}^{+}+\mathbf{A}_{\nu} \mathbf{Y}_{\mu}^{+}+\mathbf{B}_{\mu} \mathbf{Y}_{\nu}^{-}+\mathbf{B}_{\nu} \mathbf{Y}_{\mu}^{+}\right), \\
& \tilde{B}_{\mu \nu}^{\prime}=\tilde{B}_{\mu \nu}+\frac{1}{H_{u u}}\left(-\mathbf{A}_{\mu} \mathbf{Y}_{\nu}^{+}+\mathbf{Y}_{\mu}^{+} \mathbf{A}_{\nu}+\mathbf{B}_{\mu} \mathbf{Y}_{\nu}^{-}-\mathbf{Y}_{\mu}^{-} \mathbf{B}_{\nu}\right) .
\end{aligned}
$$

This result proves the fact that there are many different non-relativistic T-dual background which are related by redefinition of the Lagrange multiplicators $\lambda^{+}, \lambda^{-}$. In particular, if we choose

$$
\mathbf{Y}_{\tilde{u}}^{+}=-\frac{1}{2 \tau_{u}}, \quad \mathbf{Y}_{i}^{+}=\frac{1}{2 \tau_{u} \tau_{u}}\left(\tau_{i} H_{u u}-H_{i u} \tau_{u}-B_{i u} \tau_{u}\right), \quad \mathbf{Y}_{\mu}^{-}=0
$$

we obtain following form of the background fields

$$
\begin{array}{ll}
\tilde{G}_{\mu \tilde{u}}^{\prime}=0, & \tilde{G}_{i j}^{\prime}=G_{i j}+\frac{1}{\tau_{u} \tau_{u}}\left(H_{u u} \tau_{i} \tau_{j}-\left(H_{u i} \tau_{j}+H_{u j} \tau_{i}\right)\right), \\
\tilde{B}_{\tilde{u} i}^{\prime}=-\frac{\tau_{i}}{\tau_{u}}, & \tilde{B}_{i j}^{\prime}=B_{i j}+\frac{B_{u i} \tau_{j}-\tau_{i} B_{u j}}{\tau_{u}} .
\end{array}
$$

The background fields given in (4.13) agree with the background fields that were derived in [1], explicitly given in the expressions $(3.33 a)-(3.33 c)$ up to the sign factor in front of $\tilde{B}_{u i}$ given in $(3.44 c)$ which is a consequence of different convention. This is very nice consistency check of our approach.

\section{Transverse T-duality}

Finally we consider transverse T-duality transformations when we presume that the background has transverse symmetry with following transverse spatial Killing vector $p^{\mu}$ that obeys

$$
\tau_{\mu}^{A} p^{\mu}=0, \quad E_{\mu}^{A^{\prime}} p^{\mu} \neq 0 .
$$


We define coordinate system $x^{\mu}\left(x^{i}, z\right)$ adapted to $p^{\mu}$ such that $p^{\mu} \partial_{\mu}=\partial_{z}$. Then isometry defined above implies

$$
\tau_{z}^{A}=0, \quad E_{z}^{A^{\prime}} \neq 0
$$

and consequently

$$
\tau_{z}=\bar{\tau}_{z}=0, \quad H_{z z} \neq 0 .
$$

It is also clear that all background fields do not depend on $z$. As in previous section we perform replacement

$$
p_{\tilde{z}}=-T \partial_{\sigma} z, \quad p_{z}=-T \partial_{\sigma} \tilde{z}
$$

so that the constraints (2.9) have the form

$$
\begin{aligned}
\mathcal{H}_{\tau}^{T}= & \frac{1}{T}\left(k_{i}-B_{i z} p_{\tilde{z}}\right) H^{i j}\left(k_{j}-B_{j z} p_{\tilde{z}}\right)+\frac{2}{T}\left(-T \tilde{z}^{\prime}+T B_{z k} x^{\prime k}\right) H^{z i}\left(k_{i}-B_{i z} p_{\tilde{z}}\right) \\
& +\frac{1}{T}\left(-T \tilde{z}^{\prime}+T B_{z i} x^{i}\right) H^{z z}\left(-T \tilde{z}^{\prime}+T B_{z j} x^{\prime j}\right)+\frac{1}{T} p_{\tilde{z}} H_{z z} p_{\tilde{z}}-2 p_{\tilde{z}} H_{z i} x^{i}+T x^{i} H_{i j} x^{\prime j} \\
& -\left(k_{i}-B_{i \tilde{z}} p_{\tilde{z}}\right) H^{i j}\left(\lambda^{+} \tau_{j}+\lambda^{-} \bar{\tau}_{j}\right)-\left(-T \tilde{z}^{\prime}+T B_{z i} x^{i i}\right) H^{z j}\left(\lambda^{+} \tau_{j}+\lambda^{-} \bar{\tau}_{j}\right) \\
& +T x^{i i}\left(\lambda^{+} \tau_{i}-\lambda^{-} \bar{\tau}_{i}\right)+\frac{T}{4}\left(\lambda^{+} \tau_{i}+\lambda^{-} \bar{\tau}_{i}\right) H^{i j}\left(\lambda^{+} \tau_{j}+\lambda^{-} \bar{\tau}_{j}\right) \\
\mathcal{H}_{\sigma}^{T}= & p_{i} x^{i}+p_{\tilde{z}} \tilde{z}^{\prime} .
\end{aligned}
$$

Then following the same procedure as in previous section we find the Lagrangian in the form

$$
\begin{aligned}
\mathcal{L}^{T}= & \frac{T}{4 N}\left(\tilde{g}_{\tau \tau}-2 N^{\sigma} \tilde{g}_{\tau \sigma}+\left(N^{\sigma}\right)^{2} \tilde{g}_{\sigma \sigma}^{2}\right)-N T \tilde{g}_{\sigma \sigma}-T \partial_{\tau} \tilde{x}^{\mu} \tilde{B}_{\mu \nu} \partial_{\sigma} \tilde{x}^{\nu} \\
& +\frac{T}{2} \lambda^{+}\left(\partial_{\tau} \tilde{x}^{\mu} \tau_{\mu}-N^{\sigma} \partial_{\sigma} \tilde{x}^{\mu} \tau_{\mu}-2 N \partial_{\sigma} \tilde{x}^{\mu} \tau_{\mu}\right) \\
& +\frac{T}{2} \lambda^{-}\left(\partial_{\tau} \tilde{x}^{\mu} \bar{\tau}_{\mu}-N^{\sigma} \partial_{\sigma} \tilde{x}^{\mu} \bar{\tau}_{\mu}+2 N \partial_{\sigma} \tilde{x}^{\mu} \bar{\tau}_{\mu}\right),
\end{aligned}
$$

where again

$$
\tilde{g}_{\alpha \beta}=\tilde{H}_{\mu \nu} \partial_{\alpha} \tilde{x}^{\mu} \partial_{\beta} \tilde{x}^{\nu},
$$

where $\tilde{x}^{\mu}=\left(x^{i}, \tilde{z}\right)$ and where

$$
\begin{aligned}
\tilde{H}_{i j}=H_{i j}-\frac{H_{i z} H_{z j}}{H_{z z}}-\frac{B_{i z} B_{z j}}{H_{z z}}, & \tilde{H}_{i \tilde{z}}=\frac{B_{i z}}{H_{z z}}, & \tilde{H}_{\tilde{z} \tilde{z}}=\frac{1}{H_{z z}}, \\
\tilde{B}_{i j}=B_{i j}+\frac{B_{z i} H_{z j}-H_{i z} B_{z j}}{H_{z z}}, & \tilde{B}_{i \tilde{z}}=\frac{H_{i z}}{H_{z z}}, & \tilde{B}_{z i}=-\frac{H_{z i}}{H_{z z}} .
\end{aligned}
$$

In other words, the background fields $\tau_{\mu}$ and $\bar{\tau}_{\mu}$ do not change. The form of the Lagrangian density given above suggests that the transverse T-duality maps non-relativistic string to the non-relativistic string in T-dual background where the background fields are given by standard Buscher's rules (5.8) . In order to see that (5.6) describes non-relativist string let us solve the equations of motion for $\lambda^{+}$and $\lambda^{-}$

$$
\tau_{\tau}-N^{\sigma} \tau_{\sigma}-2 N \tau_{\sigma}=0, \quad \bar{\tau}_{\tau}-N^{\sigma} \bar{\tau}_{\sigma}+2 N \bar{\tau}_{\sigma}=0,
$$


where $\tau_{\alpha} \equiv \tau_{\mu} \partial_{\alpha} \tilde{x}^{\mu}, \bar{\tau}_{\alpha}=\bar{\tau}_{\mu} \partial_{\alpha} \tilde{x}^{\mu}$. Taking the sum and difference of these two equations we obtain two equations

$$
N^{\sigma} \tau_{\sigma}^{0}+2 N \tau_{\sigma}{ }^{1}=\tau_{\tau}^{0}, \quad N^{\sigma} \tau_{\sigma}{ }^{1}+2 N \tau_{\sigma}^{0}=\tau_{\tau}^{1}
$$

that can be solved for $N$ and $N^{\sigma}$ as

$$
N^{\sigma}=\frac{\tau_{\tau \sigma}}{\tau_{\sigma \sigma}}, \quad N=\frac{1}{2} \frac{\sqrt{-\operatorname{det} \tau_{\alpha \beta}}}{\tau_{\sigma \sigma}} .
$$

Inserting (5.11) into (5.6) and using (5.9) we obtain Lagrangian density in the form

$$
\mathcal{L}^{T}=-\frac{T}{2} \sqrt{-\operatorname{det} \tau_{\alpha \beta}} \tau^{\alpha \beta} \tilde{g}_{\alpha \beta}-B_{\mu \nu}^{\prime} \partial_{\tau} \tilde{x}^{\mu} \partial_{\beta} \tilde{x}^{\nu}
$$

where $\tau^{\alpha \beta}$ is inverse to $\tau_{\alpha \beta}$. This form of Lagrangian density corresponds to the nonrelativistic string action [17] in the background fields given in (5.8) which is again in agreement with [1].

\section{Acknowledgments}

This work was supported by the Grant Agency of the Czech Republic under the grant P201/12/G028.

Open Access. This article is distributed under the terms of the Creative Commons Attribution License (CC-BY 4.0), which permits any use, distribution and reproduction in any medium, provided the original author(s) and source are credited.

\section{References}

[1] E. Bergshoeff, J. Gomis and Z. Yan, Nonrelativistic string theory and T-duality, JHEP 11 (2018) 133 [arXiv: 1806.06071] [INSPIRE].

[2] T. Harmark et al., Strings with non-relativistic conformal symmetry and limits of the AdS/CFT correspondence, JHEP 11 (2018) 190 [arXiv:1810.05560] [INSPIRE].

[3] T. Harmark, J. Hartong and N.A. Obers, Nonrelativistic strings and limits of the AdS/CFT correspondence, Phys. Rev. D 96 (2017) 086019 [arXiv:1705.03535] [INSPIRE].

[4] J. Gomis and H. Ooguri, Nonrelativistic closed string theory, J. Math. Phys. 42 (2001) 3127 [hep-th/0009181] [INSPIRE].

[5] U.H. Danielsson, A. Guijosa and M. Kruczenski, IIA/B, wound and wrapped, JHEP 10 (2000) 020 [hep-th/0009182] [INSPIRE].

[6] T. Banks, W. Fischler, S.H. Shenker and L. Susskind, $M$ theory as a matrix model: a conjecture, Phys. Rev. D 55 (1997) 5112 [hep-th/9610043] [INSPIRE].

[7] L. Susskind, Another conjecture about M(atrix) theory, hep-th/9704080 [INSPIRE].

[8] N. Seiberg, Why is the matrix model correct?, Phys. Rev. Lett. 79 (1997) 3577 [hep-th/9710009] [INSPIRE]. 
[9] A. Sen, D0-branes on $T^{n}$ and matrix theory, Adv. Theor. Math. Phys. 2 (1998) 51 [hep-th/9709220] [INSPIRE].

[10] S. Hellerman and J. Polchinski, Compactification in the lightlike limit, Phys. Rev. D 59 (1999) 125002 [hep-th/9711037] [INSPIRE].

[11] E. Alvarez, L. Álvarez-Gaumé and Y. Lozano, A canonical approach to duality transformations, Phys. Lett. B 336 (1994) 183 [hep-th/9406206] [INSPIRE].

[12] E. Alvarez, L. Álvarez-Gaumé and Y. Lozano, An introduction to T duality in string theory, Nucl. Phys. Proc. Suppl. 41 (1995) 1 [hep-th/9410237] [InSPIRE].

[13] J. Klusoň, Nonrelativistic string theory $\sigma$-model and its canonical formulation, Eur. Phys. J. C 79 (2019) 108 [arXiv:1809.10411] [INSPIRE].

[14] J. Klusoň, Hamiltonian for a string in a Newton-Cartan background, Phys. Rev. D 98 (2018) 086010 [arXiv: 1801.10376] [INSPIRE].

[15] T.H. Buscher, A symmetry of the string background field equations, Phys. Lett. B 194 (1987) 59 [INSPIRE].

[16] T.H. Buscher, Path integral derivation of quantum duality in nonlinear $\sigma$-models, Phys. Lett. B 201 (1988) 466 [INSPIRE].

[17] R. Andringa, E. Bergshoeff, J. Gomis and M. de Roo, 'Stringy' Newton-Cartan gravity, Class. Quant. Grav. 29 (2012) 235020 [arXiv:1206.5176] [INSPIRE]. 